\title{
Thinking of Inheritance and Protection of Khoomei Art in the Mongol Nationality
}

\author{
Qi Guangtan
}

School of Music, Baicheng Normal University

Keywords: Protection and heritage of Khoomei art of the Mongol Nationality

\begin{abstract}
Khoomei is an ancient guttural singing art popular in the Mongolian nation. It has been successfully listed as an intangible cultural heritage project of humankind. The protective measures adopted for protecting Khoomei culture are not only protecting the national unique culture of our country, but also strengthening the cultural strength of our country and enhancing its international cultural competitiveness. It is of great significance to strengthen the protection and inheritance of Khoomei art and to explore its historical and cultural origins and values.
\end{abstract}

\section{Introduction}

Mongolian Khoomei art is an ancient and unique folk traditional art; its main feature is that one person's throat can issue two or more sounds with different pitches simultaneously. This unique method of uttering sounds has aroused the concern and research of many ethnic music lovers. But in terms of the current situation, it is not difficult to find that few people really understand the Khoomei culture, which does not keep in line with the protection and inheritance idea of intangible cultural heritage. Therefore, research of Khoomei culture and work of professional music workers is a great challenge.

\section{Inheritance of Mongolian Khoomei Culture}

Khoomei culture is an exquisite cultural heritage of the Mongolian people, but we have not taken protective measures in the inheritance and development of the Khoomei culture. Some countries in the Mongolian steppe region have already included Khoomei culture into the soul of the country and also regarded it as a national treasure, and inherit it

Khoomei culture was first originated in the Mongol Nationality; it is the main form adopted by herdsmen in singing. Its main characteristics is that they sing through the organ of uttering voices and can sing two vocal parts simultaneously, thus forming the rare multi-part form. It is a rare "guttural art" in the world and is reputed as "fossil of Mongolian folk music". The legend goes that the origin of Khoomei culture is from the startling and grand sounds between the waterfall and the valley. People keep imitating it and finally form the unique music model, which is the artistic form later called Khoomei.

The chanting mode of Khoomei culture is mainly the creation taking the natural scenery, landscape, animals and plants in the nature as the inspiration. Seen from the lyrics of Khoomei cultural heritage passing down from the history, Khoomei culture focuses on the short tune in singing. Despite some long-tune poems, the type is not common. Therefore, it can be concluded that in terms of the tunes of Khoomei art as well as the subjects and contents of the songs, the Khoomei singing way adopted by Inner Mongolia in the historical period of hunting in forest is the "guttural" way.

The development of Khoomei culture in Inner Mongolia has not got the attention of the nation and the state in the historical development process; it has been only spread among the folk and its development met many obstacles. So many people ignored it. A nation's culture is the basis of a country's culture. Khoomei cultural has encountered a lot of obstacles in the process of its heritage development. It is very urgent to rescue Khoomei and few singers that can act as the inheritors. 
Khoomei culture has almost disappeared for more than 100 years in Inner Mongolian grassland area in China. It now faces the possibility of disappearance, which has caused a serious threat to the Khoomei cultural heritage. Therefore, in order to save the crisis of the Chinese culture, well protecting the national culture is a country's spiritual civilization, which has an important significance in the development of culture, which is our duty and responsibility!

\section{Current Development Status of Khoomei Culture}

It is not an easy thing for Khoomei culture to develop till today and become China's national cultural heritage. Some ambitious people in the Inner Mongolia Arts Festival people have kept studying, learning and disseminating Khoomei culture and have achieved certain good results.

Since the Khoomei art culture has been listed as a human intangible cultural heritage, people began to have more understanding of this cultural art, and also began to keep contacting learning. Other countries where Khoomei spreads have also begun to attach importance to the protection and inheritance of Khoomei culture.

Now it has become a popular culture and music market; many foreign-style jazzes have begun to penetrate into our cultural traditions. The spread influence of this culture has two aspects. On the one hand, it can promote the development of our culture, making more culture enter people's lives, and get development and dissemination; but at the same time, the spread of many foreign cultures in China will make a lot of people begin to ignore the domestic cultural heritage; they do not know how to take its essence and remove its dross, while ignoring the development and inheritance of domestic local culture.

With the continuous development of Khoomei art, people have begun to make innovations of Khoomei. The performance forms of Khoomei cultural art tend to diversify and are gradually integrated into the jazz and other modern art culture. For the Mongols, their horses and wrestling and other large-scale activities are highly emphasized; Khoomei culture has begun to integrate into it. The number of people who learn Khoomei culture is gradually increased. According to statistics, now nearly 300 people have been able to master the Khoomei culture and play alone. In order to allow more people to contact and understand the Khoomei culture, Khoomei classroom teaching has been opened in the university classes and other institutions.

The Inner Mongolian opera and music culture has always been regarded as a national cultural heritage of our country for heritage and development, and Inner Mongolian national culture chorus is an artistic and cultural group with distinctive national features. It has achieved excellent results in a few international cultural competitions and has won several awards. It has enjoyed a high reputation in the international arena. In recent years, they begin to study the Khoomei cultural singing that has been lost for a long time, which, after all, is a major feature of our culture. It has also won many concerns in the international community. The singing method of Khoomei culture has been used for many times at home and abroad and has won favor from the majority of the audience.

\section{Protection of Khoomei Culture}

Since the reform and opening up, the grassland cultural environment has suffered from a great impact and the state began to require a large number of herdsmen to reclaim land. Due to the changes of the cultural environment and living environment, Khoomei singing way began to be ignored. It has several masters, who are merely singing for livelihood; their styles have been different from the Khoomei style. At present, the popularity of the pop culture is a great impact on the Khoomei culture and there are few Khoomei's successors.

Khoomei culture, as a cultural heritage should combine protection and development and develop under the premise of protection. It should be kept protecting in the development, to avoid the loss and deterioration in the process of cultural development. The intangible cultural heritage is a witness to the historical development of the region and the main manifestation of the local characteristics in the Inner Mongolia region. It plays a key role in publicizing the local civilization 
in the future and is is of great significance to the promotion and inheritance of the local cultural heritage.

Khoomei culture is as a local cultural heritage. While protecting it, the artistic modes of cultural centers and cultural stations can be used to popularize it. Carry out the collective activities such as masses' learning and singing of Khoomei, so as to make more people feel the charm of Khoomei culture, cultivate more people who appreciate the Khoomei culture so as to to continue to learn and inherit. Combine the protection of Khoomei culture and the development of national culture, so as to enhance the community's awareness of protecting the intangible cultural heritage of Khoomei. Besides, the government can also play a role in calling on more people to pay attention to Khoomei culture. Inner Mongolia has rich tourism resources, so the tourism projects focusing on grassland nomadic can be constructed to attract more tourists, integrate the Khoomei culture into the tourism projects, so as to both develop the economy of Inner Mongolia, but also to promote the protection and promotion of Khoomei art, so that more people can understand Khoomei, joined the work of Khoomei art protection. Purposefully and consciously regard the popularity of the Khoomei culture as an important mass activity in the Mongolian region and attract everyone's attention.

In addition, in some cultural and artistic festivals, or the daily singing, focus on making Khoomei culture singing popular and further reinforce people's foundation of the Khoomei culture and bring the Khoomei culture back to the depths of the grassland art and to the herders. Cultivate more participants and singers for Khoomei art and ask more people from the grassland to spread Khoomei culture to the world, so as to people re-recognize the charm of Khoomei culture, thereby enhancing the national protection and heritage awareness of Khoomei culture and silently bringing the Khoomei culture into the blood of the nation.

The development of a country and the nation are inseparable from the development of national culture. Culture is the soul and fundamental of a nation. Culture is the unique, valuable priceless crystal of material and non-material in the process of human development. Khoomei culture, as a Mongolian cultural characteristics, is also the cultural characteristics of the Chinese nation and is the symbol of culture prosperity of our nation. It is also the logo for our national culture to the world, so as to promote the common development of national culture. Eliminate the poor cultures.

Khoomei culture has been widely welcomed and loved in the world. The Chinese nation has the history of more than five thousand years. Its cultural essence and prosperity are also widely recognized in the world. To increase the protection and the inheritance of Khoomei culture can enhance the cultural soft power of our country. The competition among the big powers in the world tends to be the invasion of cultural strength. In face of the invasion of the foreign cultures, our country can absorb it rather than lose orientations.

\section{Conclusion}

China is a multi-ethnic multi-cultural united country. Therefore, China has a rich cultural background and cultural contents. These cultures have the immeasurable values of the study of the development conditions of the nationalities in our country. Each of us has the obligation to protect and develop the remaining cultures exclusive to our country and make it keep growing.

Out of the number of people and living environment of the minorities, the development and inheritance of its national culture may face challenges to a certain extent, so our national government needs to help the cultural essence of ethnic minorities to be inherited and developed, so as to make people of 56 nationalities in our country realize that the inheritance and development of culture is not only a responsibility of our own, but also a task. Make each of our nation accept own national culture, integrate it into their lives and cultivate the qualities of national culture of the next generations. Enable them to contact the inherited cultures of their nations, accept the influences of the cultures, cultivate the interests and sense of responsibility of can be integrated into the life of the positive to cultivate their next generation of national cultural the next generation, keep strengthening their sense of honor and mission. Let them recognize the great historical value and historical significance of their own culture, so that they will intentionally or unintentionally reveal the flavor of ethnic minorities in their daily life, and make other nations have a sense of mystery of 
their own national cultures and feel the historical atmosphere of the traditional culture.

At present, there are many excellent cultures in our country that are not protected and inherited. We should actively face these problems, especially the cultures of ethnic minorities. We should carry out inheritance and development of the cultures together to prevent the loss of outstanding national cultures in the process of development.

\section{References}

[1] Su Bude, Plan of "Application Research of Mongolian Traditional Music in Early Childhood Education in the First Half of 2008"[A]; Result Set of Eleventh Five-stage of National Teachers Research Fund (Inner Mongolia volume) [C]; 2013

[2] Su Bude, Implementation Plan of Application Research of Mongolian Traditional Music in Early Childhood Education[A]; Result Set of Eleventh Five-stage of National Teachers Research Fund (Inner Mongolia volume) [C]; 2014

[3] Su Bude, Final-term Experiment Report of Application Research of Mongolian Traditional Music in Early Childhood Education[A]; Result Set of Eleventh Five-stage of National Teachers Research Fund (Inner Mongolia volume) [C]; 2015

[4] Liu Ruixia, Embodiment of Grassland Culture Tradition in the Mongolian Style Piano Works[J]; Popular Literature; Periodical 03, 2014

[5] Liu Xinhe, Several Problems of Research of Mongolian Songs[J]; Inner Mongolia Art; Periodical 02, 2012

[6] Ma Shuhao, National Characteristics of Mongolian Piano Works[J]; Drama Home (First Half); Periodical 02, 2013

[8] Su Jina, Development of Mongolian-style Piano Works[J]; Journal of Inner Mongolia University for the Nationalities (Social Science Edition); Periodical 03, 2008

[9] Li Shurong, Cultural Consciousness Contained in "Characteristics"--Comment on Original Large Mongolian Theme Dance Auspicious Grassland[J]; Journal of Art College of Inner Mongolia University; Periodical 01, 2008 